\title{
Pollution characteristics and ecological risk of polycyclic aromatic hydrocarbons (PAHs) in surface sediments of the southern part of the Haihe River system in China
}

\author{
LIU Feng, LIU JingLing* , CHEN QiuYing, WANG BinBin \& CAO ZhiGuo \\ State Key Joint Laboratory of Environmental Simulation and Pollution Control, School of Environment, Beijing Normal University, Beijing \\ 100875, China
}

Received August 15, 2012; accepted January 7, 2013; published online March 7, 2013

\begin{abstract}
The southern part of the Haihe River system, the largest river system in the Haihe River Basin, is important to maintain a healthy ecosystem and safe drinking water as well as for the economic development of China. To assess the effect of rapid industrialization and urbanization on the pattern of polycyclic aromatic hydrocarbon (PAH) contamination, the PAH pollution characteristics of the southern part of the Haihe River system was investigated. Sixteen PAHs in surface sediments samples collected from 24 sites covering the southern part of the Haihe River system from the upstream to the estuaries were detected and analyzed. The total PAH concentration ranged from 258.77 to $11296.66 \mathrm{ng} / \mathrm{g}$ dry weight. On average, 2-, 3-, 4-, 5- and 6-ring PAHs comprised $25.32 \%, 27.22 \%, 22.62 \%, 14.89 \%$ and $9.96 \%$ of the total PAHs, respectively. Sites with high concentrations were concentrated in the Fuhe River, Fuyang River and Wei River, which are located near cities. A risk quotient (RQ) was used to assess the ecological risk of PAHs. The mean values of the $\mathrm{RQ}_{(\mathrm{MPC})}$ of individual PAHs were lower than 1.00, except for those of naphthalene (2.00) and pyrene (1.67). The $\mathrm{RQ}_{\Sigma \mathrm{PAH}}$ calculated for the samples indicated that the Xidayang reservoir and the estuary of the Ziya River were determined to be at a low risk level, while Baoding City, Handan City and Aixinzhuang were determined to be at a high risk level.
\end{abstract}

polycyclic aromatic hydrocarbons, surface sediments, the southern part of the Haihe River system, distribution, composition profile, ecological risk assessment

Citation: Liu F, Liu J L, Chen Q Y, et al. Pollution characteristics and ecological risk of polycyclic aromatic hydrocarbons (PAHs) in surface sediments of the southern part of the Haihe River system in China. Chin Sci Bull, 2013, 58: 3348-3356, doi: 10.1007/s11434-013-5677-6

Polycyclic aromatic hydrocarbons (PAHs) are persistent organic pollutants and have been listed as priority pollutants by both the US Environmental Protection Agency and the European Union due to their toxicity, carcinogenicity and mutagenicity [1]. PAHs are known to enter the aquatic environment through industrial and urban discharge, petroleum spills, fossil fuel combustion and atmospheric deposition [2]. Due to their lipophilicity, PAHs in water tend to accumulate in bottom sediments [3], which acts as a longterm reservoir [4]. PAHs also can bio-accumulate through the food chain [5], and the exposure of humans to PAHs may enhance the risk of cancer and other adverse health

*Corresponding author (email: jingling@bnu.edu.cn) effects [6]. Therefore, the accumulation of PAHs in sediments has received much attention [7,8]. Existing research on PAHs in Haihe River Basin has mainly focused on a few important reaches, such as rivers in Tianjin and Beijing $[9,10]$. Research focusing on other river systems in Haihe River Basin has rarely been reported. Only a few studies about PAHs in the Tuhai-Majia River [11], Luan River [12] and Zhangweinan River [13] have been reported. Previously, there were no integrated and detailed studies on PAHs in surface sediments of the southern part of the Haihe River system.

The southern part of the Haihe River system is the largest of the four river systems in Haihe River Basin, with an area of $1.24 \times 10^{5} \mathrm{~km}^{2}$. The Haihe River system includes some 
big cities, such as Shijiazhuang City, Handan City, Xingtai City and Baoding City. It is mainly composed of three rivers named Qaqing River, Ziya River and Zhangweinan River. With the growth of the population and economy, intense land utilization and water resources development has occurred. The contamination of this river system has been aggravated by the increased discharge of industrial, agricultural and urban wastewater. However, there are ten water resource fields used for drinking and transportation in the southern part of the Haihe River system, so the level of PAHs in this water system are closely related to the safety of the drinking water. However, no research specifically focusing on the PAHs pollution levels in this river system have been reported. Because sediments are the ultimate sink and reservoir of PAHs, identifying the pollution characteristics of PAHs in the surface sediments of the southern part of the Haihe River system represents important work.

The aims of this study are (i) to investigate the concentrations and distribution of PAHs in superficial sediments from the southern part of the Haihe River system and (ii) to evaluate the ecological risk of PAHs using a risk quotient (RQ). To achieve these goals, sediment samples were collected from locations ranging from upstream to estuaries in the southern part of the Haihe River system. Sixteen PAHs, listed as United States Environmental Protection Agency (USEPA) priority pollutants, were investigated.

\section{Materials and methods}

\subsection{Sampling}

The sampling sites are shown in Figure 1. Among the 24 sampling stations, 7 were located in suburban areas, 6 in cities, 6 in reservoirs and 3 in estuaries. Samples from suburban areas that were not under the direct impact of industrial and traffic activities could reflect the "background" level of the PAHs in the study area, while sites close to cities could reflect the "maximum" value and the effect on PAHs contamination caused by rapid industrialization and urbanization. Water resource areas, natural reservation regions and estuaries were the most important and sensitive areas in the basin. Thus, sampling sites in these areas were essential for the management and maintenance of safe drinking water in the southern part of the Haihe River system.

Samples of surface sediments were collected in May 2010. Global positioning system (GPS) was used to locate the sampling sites. Surface sediment samples $(1-5 \mathrm{~cm}$ deep) were collected with a grab sampler, and the top $1 \mathrm{~cm}$ of the surface layer was carefully removed with a stainless steel spoon [14] and stored in aluminum jars that had been prewashed with methylene chloride. The samples were cooled on ice during transportation to the laboratory, where they were stored at $-20^{\circ} \mathrm{C}$ before being freeze-dried. The dried sediment samples were sieved to obtain particles of less than $180 \mu \mathrm{m}$ [15]. At each site, 3 samples were collected

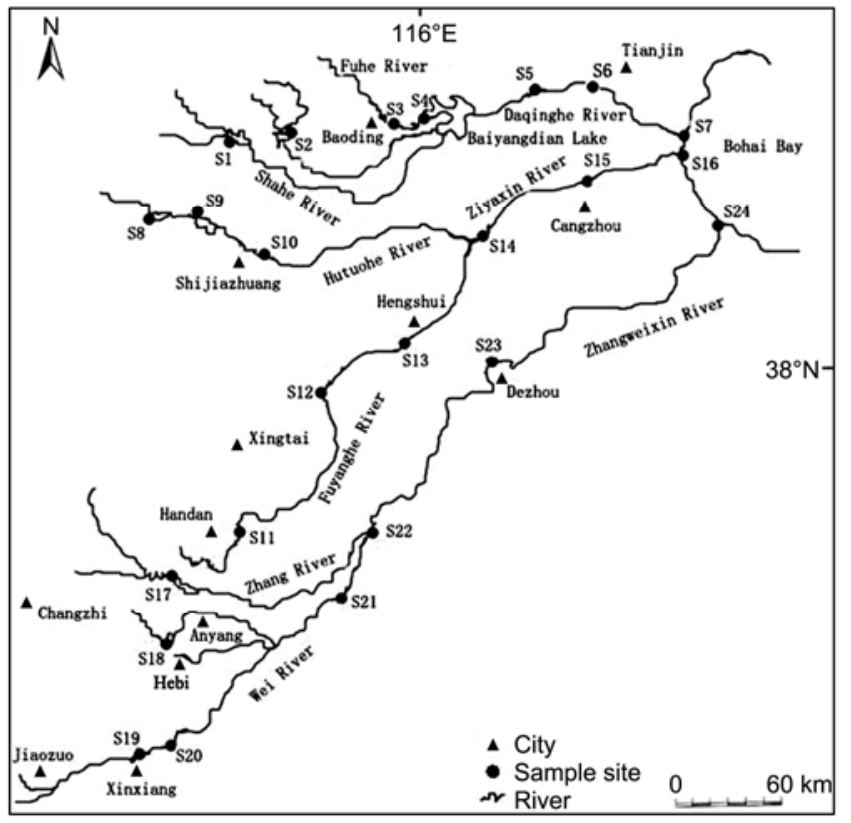

Figure 1 Location and sampling sites of the southern part of the Haihe River system. S1: Wangkuai; S2: Xidayang; S3: Baoding; S4: Fuhe; S5: Bazhou; S6: Jinghai; S7: Estuary of Duliujian river; S8: Gangnan; S9: Huangbizhuang; S10: Shijiazhaung; S11: Handan; S12: Aixinzhuang; S13: Hengshui Lake; S14: Xianxian; S15: Cangzhou; S16: Estuary of Ziyaxin River; S17: Yucheng; S18: Xiaonanhai; S19: Xinxiang; S20: Weihui; S21: Longwangmiao; S22: Guantao; S23: Dezhou; S24: Estuary of Zhangweinan River.

within a distance of $5 \mathrm{~m}$ [16]. Triplicate samples were mixed thoroughly to make a composite sample to minimize withinsite variability and ensure that the sediment samples collected were representative of each site [17].

S1 to S7 were located in the Daqing River. S1 and S2 were located in reservoirs, while S3 was located in Baoding City and was directly affected by the wastewater of the city. S4 was located in the Fuhe River at the entrance to Baiyangdian Lake, which is a natural reservoir. S5 and S6 were located in suburban areas, and S7 was located in a port. Nine sampling sites (S8-S16) were located in the Ziya River, of which S13 was located in a natural reservoir named Hengshui Lake. S14 was at the confluence of the Fuyanghe River and the Hutuohe River, and S16 was in the estuary. The Zhangweinan River had 8 sampling sites (S17-S24). Of these 8 sites, S17 and S18 were located in two reservoirs, S19 and S23 were close to Xinxiang City and Dezhou City, S20 and S22 were located in suburban areas and S24 was in the estuary of the Zhangweinan River.

\subsection{Sample extraction}

Frozen sediment samples were dried using a freeze-drying apparatus. Prior to extraction, these samples were ground into granular powder that was passed through a 100-mesh sieve and homogenized. Mashed samples were first mixed with anhydrous sodium sulfate in a weight ratio of 5:1 and 
with activated copper sheets in a ratio of $1: 1$, to desulfurize the extract. The mixtures were extracted by accelerated solvent extraction (ASE300, Dionex, Sunnyvale, CA) with $n$ hexane/dichloromethane $(1: 1, \mathrm{v} / \mathrm{v}$ ) for $10 \mathrm{~min}$ (two static cycles). The extraction temperature was set at $125^{\circ} \mathrm{C}$ and pressure at $1500 \mathrm{~Pa}$. The extracts were reduced to about 2 $\mathrm{mL}$ using a rotary evaporator and purified using a silica gel column packed with anhydrous sodium sulfate. The column was first rinsed with $10 \mathrm{~mL} n$-hexane solvent to remove non-PAH impurities, such as aliphatic hydrocarbons. Then, the column was rinsed with $15 \mathrm{~mL}$ of mixed dichloromethane and $n$-hexane $(1: 1, \mathrm{v} / \mathrm{v})$. The second eluate contained the PAHs and was collected and concentrated using a rotary evaporator. The effluent was then reduced to about $0.5 \mathrm{~mL}$ under a stream of purified $\mathrm{N}_{2}$ in $\mathrm{KD}$ vessels and adjusted to $1.0 \mathrm{~mL}$ with hexane for GC-MS analysis.

\subsection{PAHs analyses}

All of the extracted samples were analyzed using a HewlettPackard 6890 Gas Chromatograph (GC) connected to a Hewlett-Packard 5973 N Mass Selective Detector (MSD) (Agilent, Wilmington, DE). The chromatographic column was a $30 \mathrm{~m}, 0.25 \mathrm{~mm}$ inner diameter, $0.25 \mathrm{~lm}$ film thickness HP-5MS capillary column (\#19091s-433, Agilent, J and W Scientific, Folsom, CA). Helium was used as the carrier gas at a constant flow rate of $1 \mathrm{~mL} / \mathrm{min}$. The oven temperature was ramped from 60 to $260^{\circ} \mathrm{C}$ at $6^{\circ} \mathrm{C} / \mathrm{min}$ and then held for $15 \mathrm{~min}$. The MSD was operated in the electron impact mode at $70 \mathrm{eV}$. The ion source temperature was $280^{\circ} \mathrm{C}$. The mass spectra were recorded at a scan mode covering the range of 45-400 mass units for sediment samples. Quantification was performed using the external standard method with a 16 PAH standard mixture (PPH-10JM, Chem Service Inc., West Chester, PA). The retention time and identity of the PAHs were also confirmed using this standard. The GC/MS data were acquired and processed using Enhanced ChemStation (HPG1701CA, Agilent). The 16 PAHs detected in sequence were the following: naphthalene (Nap), ace-naphthylene (Ace), acenaphthene (Acp), fluorene (Fl), phenanthrene (Phe), anthracene (An), fluoranthene (Flu), pyrene (Pyr), benz[a]anthracene (BaA), chrysene (Chr), benzo[b]fluoranthene $(\mathrm{BbF})$, benzo[k]fluoranthene $(\mathrm{BkF})$, benzo[a]pyrene $(\mathrm{BaP})$, indeno[1,2,3-cd] pyrene $(\mathrm{InP})$, dibenzo[a,h]anthracene (DbA), and benzo [ghi]perylene (BghiP).

\subsection{Analytical quality control}

All data were subjected to strict quality control procedures. Naphthalene-d8, acenaphthylene-d10, phenanthrene-d10, chrysene-d12 and perylene-d12 were used as surrogates, and their recoveries were $51.8 \pm 10.7 \%, 78.6 \pm 10.9 \%, 83.8 \pm$ $10.5 \%, 93.2 \pm 9.1 \%$ and $88.9 \pm 10.1 \%$, respectively. The limits of detection ranged from 1.5 to $9.3 \mathrm{ng} / \mathrm{g}$. The results showed that precision was generally satisfactory.

\section{Results and discussion}

\subsection{Concentrations and composition profiles}

The concentrations of PAHs in sediments from the 24 sampling sites are listed in Table 1. The concentration ranges of total PAHs in sediments of the Daqing River, Ziya River and Zhangweinan River were 258.77-1346.01, 326.8911296.66 and 470.88-2315.19 ng/g dry weight, respectively. For the Daqing River, Ziya River and Zhangweinan River, the mean concentrations of total PAHs of the three rivers were 1249.72, 2926.40 and $1049.30 \mathrm{ng} / \mathrm{g}$, respectively, and the median concentrations were $1184.91,1138.40$ and $719.75 \mathrm{ng} / \mathrm{g}$, respectively. The median concentrations were generally lower than the mean concentrations, suggesting that the majority of these sites contained lower concentrations than the mean. Comparing the mean and median concentrations of the total PAHs from the three rivers, it was clear that the PAHs of the Zhangweinan River were at a relatively low level. According to the water resources bulletin of Haihe River Basin (conventional pollutants) in 2010, the water quality of the Wei River and the Zhangweixin River was worse than grade V [18], and the Zhangweinan River had worse water quality than the other two rivers of the southern part of the Haihe River system. However, the levels of PAHs in the water [13] and sediments of the Zhangweinan River were not serious, so the other pollutants should be controlled first.

The concentrations of PAHs in the sediments from Haihe River Basin were much higher than those from rivers both overseas and domestic, such as Malaysian rivers, the Hyeongsan River, the Yangtze River, and the Yellow River (Table 2). The PAHs pollution in Haihe River Basin was relatively high compared to other rivers of the world. Compared with rivers within Haihe River Basin, the concentrations of PAHs in the southern part of the Haihe River system was higher than that of the Luan River and the TuhaiMajia River system but lower than that of rivers in Tianjin. As PAHs lack functional groups, they are among the most stable organic indicators, and their concentrations in sediments have accurately recorded their history input [24]. Therefore, these results could be attributed to the fact that Tianjin was an old, large and established industrial city with the largest port in north China, while the Luan River and the Tuhai-Majia River system were primarily utilized for agriculture. Thus, in Haihe River Basin, the PAH concentrations appeared to be consistent with the respective regions' degree of industrialization.

In the southern part of the Haihe River system, most sites were dominated by Nap, Phe and Flu, and the mean concentrations of the three PAHs were 267.77, 261.56 and 191.37 $\mathrm{ng} / \mathrm{g}$, respectively. However, the concentrations of Pyr, BaA, $\mathrm{Chr}, \mathrm{BbF}, \mathrm{BkF}, \mathrm{BaP}, \mathrm{InP}, \mathrm{BghiP}$ were also high at Handan 


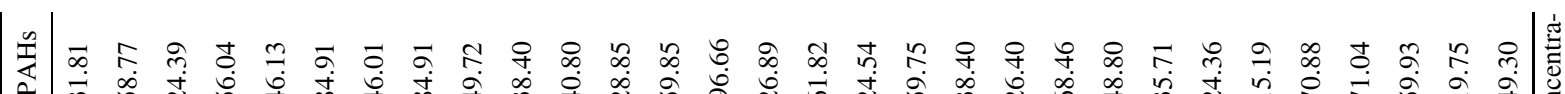

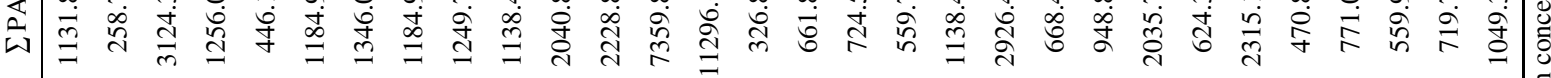

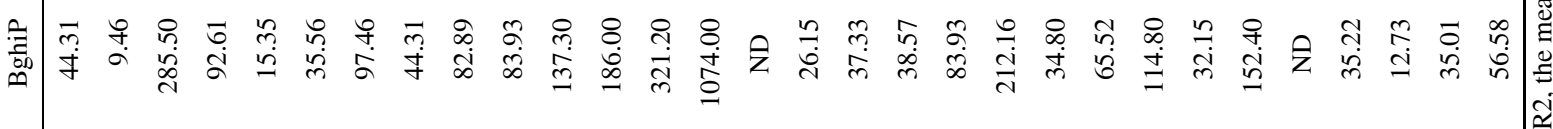

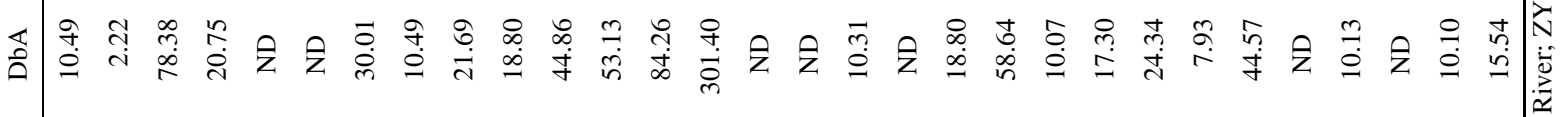

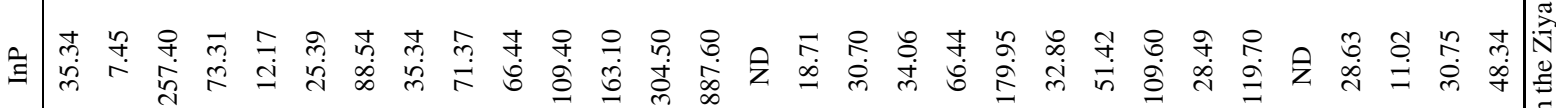

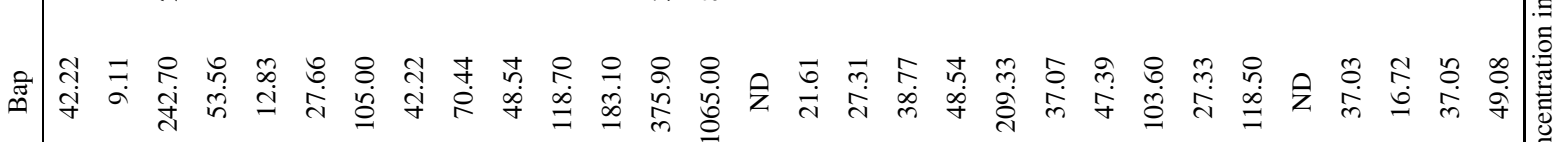

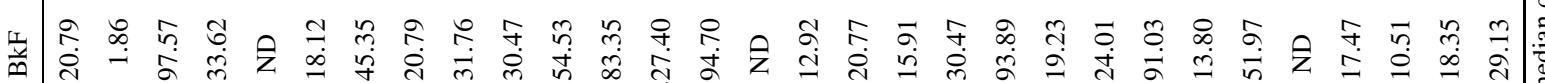

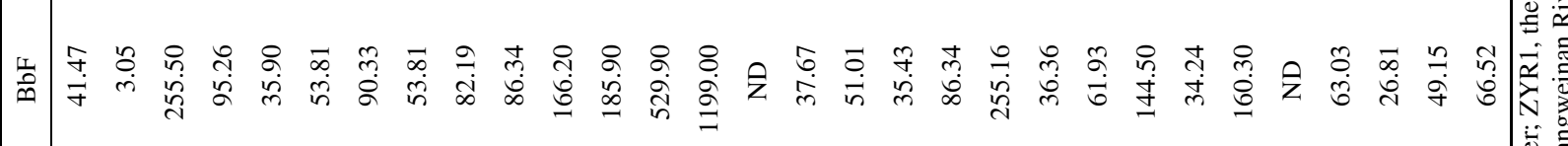

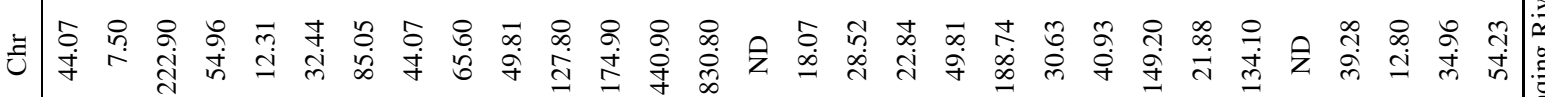

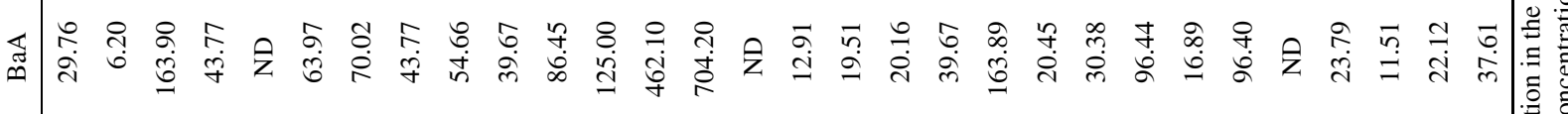

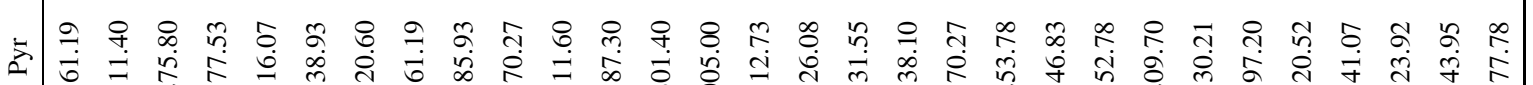

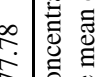

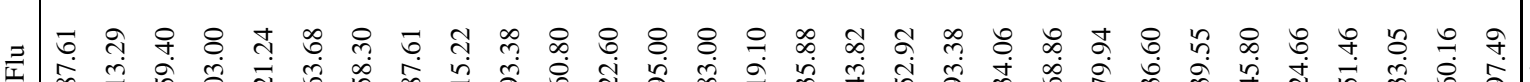

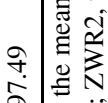

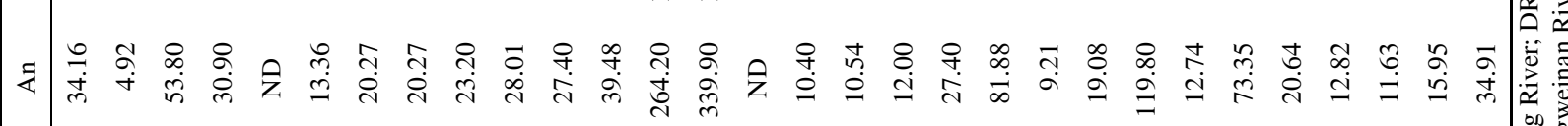

๑ 8 t

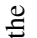

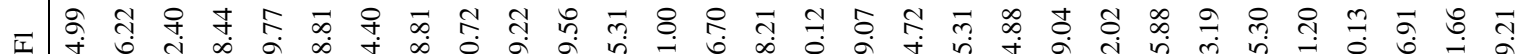

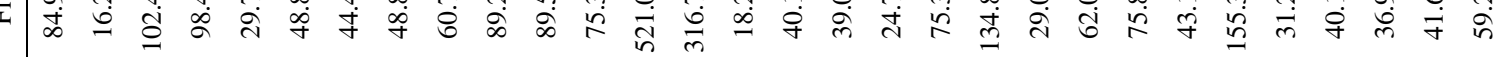

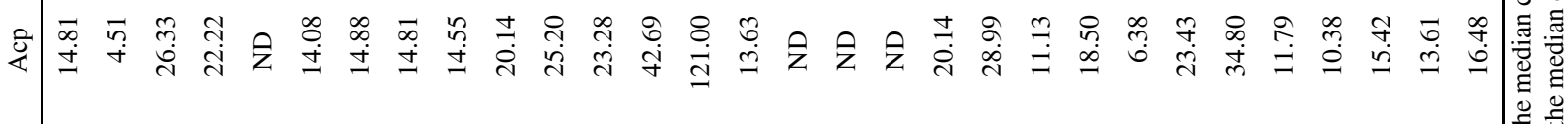

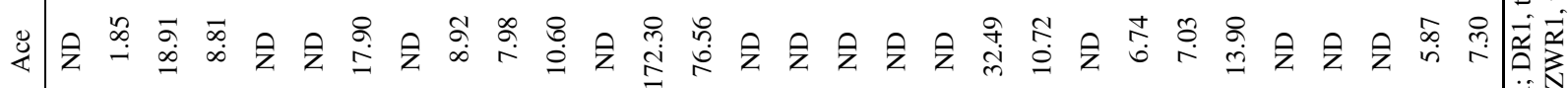

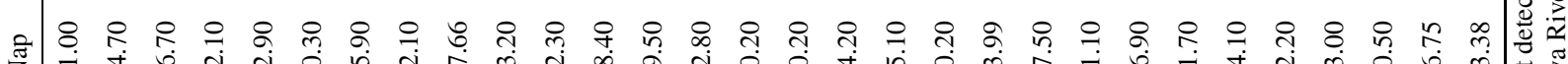
ठ্ট

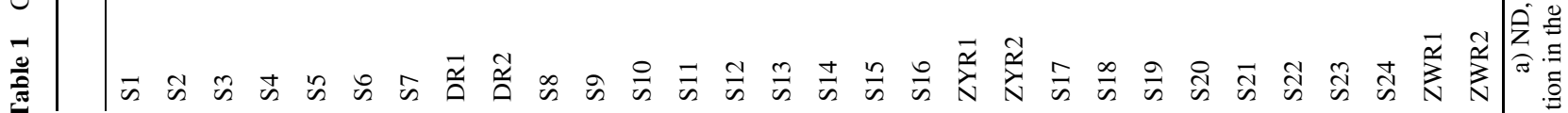


Table 2 Concentration ranges of PAHs in sediments of rivers in the world

\begin{tabular}{|c|c|c|c|c|}
\hline Region & Location & $N^{\mathrm{a})}$ & Concentration (ng/g) & Ref \\
\hline \multirow[t]{3}{*}{ Overseas } & Athabasca River, Canada & 16 & $10-34700$ & [19] \\
\hline & Malaysian rivers & 16 & $4-924$ & [20] \\
\hline & Hyeongsan River, Korea & 16 & $5.30-7680$ & [17] \\
\hline \multirow[t]{4}{*}{ Domestic } & Qiangtang River, China & 15 & $91-614$ & [21] \\
\hline & Yangtze River, China & 16 & $72.4-995.2$ & {$[22]$} \\
\hline & Yellow River, China & 16 & $31-133$ & [23] \\
\hline & Daliao River & 28 & $102.9-3419.2$ & [16] \\
\hline \multirow[t]{5}{*}{ Haihe River Basin } & Rivers in Tianjin & 16 & $787-1943000$ & [9] \\
\hline & Haihe River, Tianjin & 16 & $774.8-255371.91$ & [10] \\
\hline & Tuhai-Majia River system & 9 & $311.69-3736.32$ & [11] \\
\hline & Luan River & 20 & $20.9-287$ & [12] \\
\hline & South part of Haihe River system & 24 & $258.77-11296.66$ & This study \\
\hline
\end{tabular}

a) $N$, number of kinds of PHAs.

(S11) and Aixinzhuang (S12). The composition pattern of 16 PAHs in sediments of the southern part of the Haihe River system is shown in Figure 2. On average, 2-, 3-, 4-, 5and 6-ring PAHs comprised 25.32\%, 27.22\%, 22.62\%, $14.89 \%$ and $9.96 \%$ of the total PAHs, respectively. The measured PAHs at most stations consisted mainly of 2-, 3and 4-ring PAHs, and the composition profile was the same with the Jialu River [25], except for Baoding City (S3), the estuary of the Daqing River (S7), Shijiazhuang City (S10), Aixinzhuang (S12) and Xinxiang (S19), which were mainly composed of high molecular weight (5- and 6-ring) PAHs and were all directly or indirectly affected by cities. PAHs formed in high temperature combustion processes have a condensed structure (4-, 5- and 6-ring) and were usually identified as the majority of PAHs input from urban wastewater and industrial runoff [26]. PAHs originating from unburned products have a smaller number of rings (2and 3-ring) [16]. The predominate source of PAHs for most stations was discharging of crude oil and related products. The PAHs found at sites S3, S7, S10, S12 and S19 were mainly from industrial and urban emissions.

\subsection{Distribution}

The distribution of PAHs in sediments is shown in Figure 3. For the Daqing River, the lowest concentration was found at $\mathrm{S} 2$, the Xidayang reservoir, which was the main water source for Baoding City and one of the emergency water reserves for Beijing. The highest concentration was found at S3, which was located downstream in the Fuhe River. The Fuhe River was mainly influenced by the sewage and industrial wastewater from Baoding City [27]. The most polluted sites were found in the middle and lower reaches of the region; the main reason might be that the large city of Baoding was located in the middle reach and the municipality Tianjin was located in the lower reach. This finding was

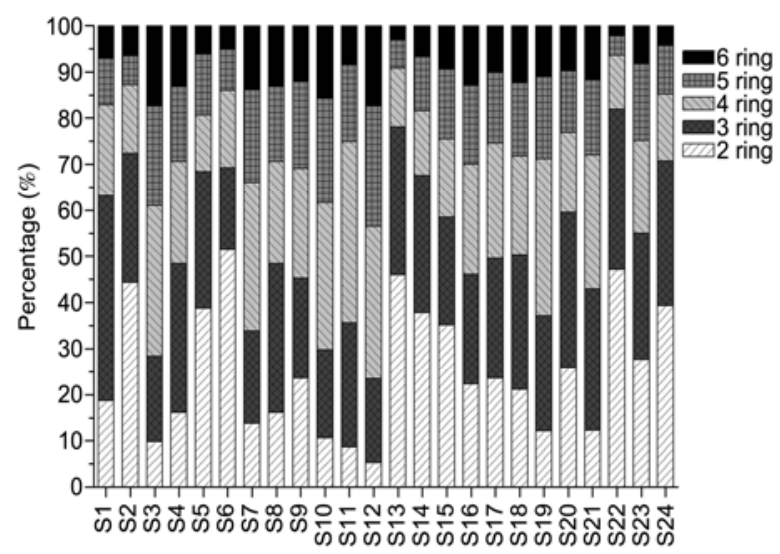

Figure 2 The percentages of PAHs of different ring numbers in surface sediments from the southern part of the Haihe River system.

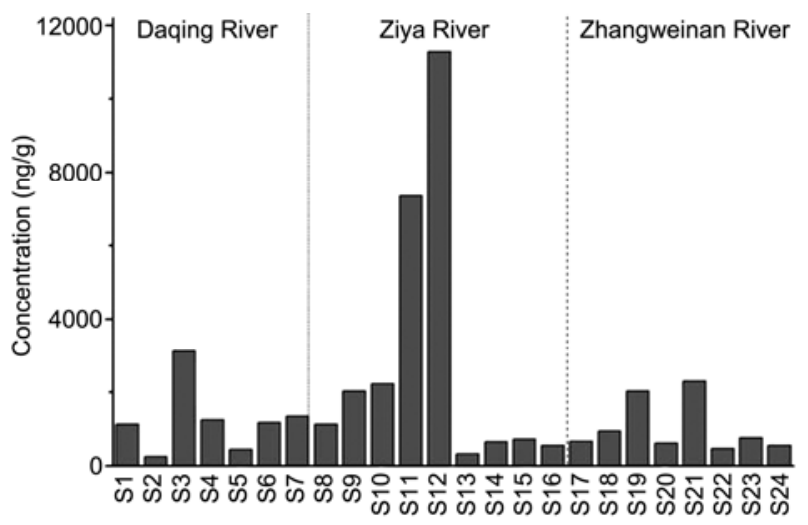

Figure 3 Distribution pattern of total PAHs in sediments from the southern part of the Haihe River system.

similar to the result derived from the Yellow River [23].

For the Ziya River, the highest concentration of PAHs was found at S12 (11296.66 ng/g), which was located in 
Aixinzhuang, which is a rural area. This finding might be because of the proximity of Xingtai City. Xingtai City is a big industrial city with an area of $12486 \mathrm{~km}^{2}$ and a population of 6.7 million. It is famous for equipment manufacturing, coal salt chemical production and auto manufacturing. Industrial waste and urban runoff might be the reason for the high concentration of PAHs in Aixinzhuang. In contrast, the concentration of total PAHs in some cities, such as Cangzhou City (724.54 ng/g) and Shijiazhuang City (2228.85 $\mathrm{ng} / \mathrm{g}$ ), were much lower. The lowest concentration was detected at Hengshui Lake (326.89 ng/g), and the second lowest was in the estuary (559.75 ng/g). Because Handan City is located upstream and is a mining city, the Fuyang River was one of the most polluted rivers in Hebei province [28]. For the Ziya River, the highly polluted sites were concentrated in the middle and upper reaches, while the lower reaches and the estuary had relatively low PAH concentrations.

For the Zhangweinan River, relatively high concentrations of PAHs were detected at S20 (Xinxiang City, $2035.71 \mathrm{ng} / \mathrm{g}$ ) and S22 (Longwangmiao, $2315.19 \mathrm{ng} / \mathrm{g}$ ), and relatively low concentrations were detected at S23 (Guantao, $470.88 \mathrm{ng} / \mathrm{g}$ ) and S25 (estuary, $559.93 \mathrm{ng} / \mathrm{g}$ ). Longwangmiao is located in the lower reach of the Wei River, and wastewater from upstream (Xinxiang City and Hebi City) resulted in the relatively high concentration of PAHs in this sampling site. Xinxiang was directly affected by its industrial emissions and traffic pollution, which caused the high concentration of PAHs. Guantao is in a rural area less affected by cities, which led to the lowest concentration of PAHs.

The concentrations of PAHs in cities were higher than that in rural areas (Figure 4) in the Daqing River and the Zhangweinan River, and the distribution pattern was similar to that in peninsular Malaysia [20] and different from the Ziya River. This finding was mainly because of the extremely high concentration observed at Aixinzhuang (11296.66 $\mathrm{ng} / \mathrm{g}$ ), and the sites in the rural areas had much lower concentrations (661.82 ng/g). Comparing the PAHs concentrations in reserves upstream with the estuaries downstream, the concentrations in the estuaries were relatively low in the Ziya River and the Zhangweinan River. For the two rivers, which have large industrial and agricultural water consumption, the volume of water flowing into the sea was small, and zero flow happened frequently in recent years [13]. The two estuaries were located in rural areas, so they had very little input of PAHs from upriver or from the cities, so the concentration of PAHs in the estuaries was relatively low. In contrast, the concentration of total PAHs in the Daqing River estuary was relatively high, which may be because this estuary was located in Tianjin and affected by petroleum spills, industrial and urban discharges.

The coefficient of variation $(\mathrm{CV})$ of total PAHs of the southern part of the Haihe River system was $138 \%(n=24)$, suggesting great variation in the concentrations of PAHs in

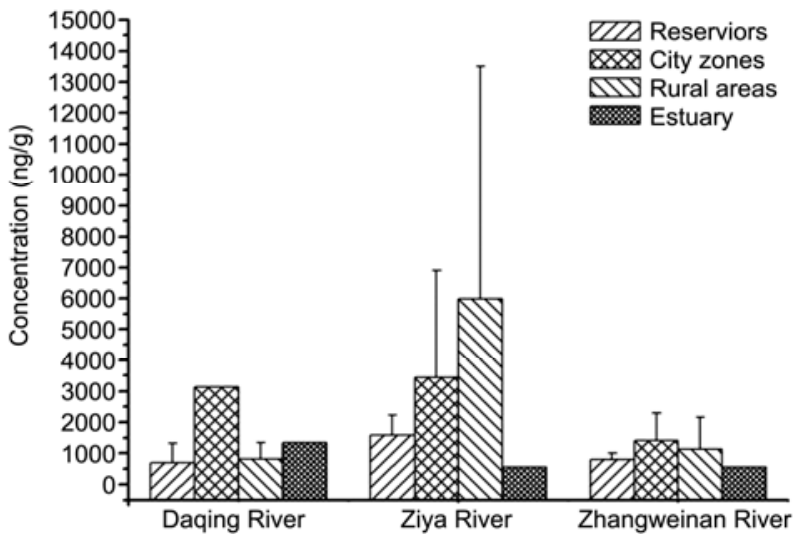

Figure 4 Contrast of the PAHs in different regions of the Daqing River, the Ziya River and the Zhangweinan River.

different sampling sites. This finding was true of the CVs of the Daqing River $(n=7, C V=74 \%)$, the Ziya River ( $n=9$, $130 \%)$ and the Zhangweinan River $(n=8, C V=68 \%)$. The highly polluted sites were concentrated in the middle and lower reaches of the Daqing River and in the upper and middle reaches of the Ziya River and the Zhangweinan River. However, the spatial distribution patterns of PAHs in the three rivers were in accordance with the distribution pattern of cities. Generally, sites located in cities exhibited higher PAHs concentrations than those in rural areas. The relatively high concentrations were closely related to the discharge of industrial wastewater, urban runoff and emission of atmospheric particles from the cities. In the southern part of the Haihe River system, the highly polluted sections were the Fuhe River, the lower reach of the Daqing River, the Fuyang River and the Wei River. These highly polluted sections were mainly impacted by Baoding City, the Tianjin municipality, Handan City, Xinxiang City and Hebi City. We suggest that the discharge of industrial waste water, urban runoffs and emission of atmospheric particles in the five cities should be controlled.

\subsection{Ecological risk assessment}

PAHs accumulated in sediments could be used by emergent macrophytes [29], such as cattail and reed, and enter aquatic food webs [30]. Therefore, the PAHs in sediments pose a potential risk for aquatic ecosystems. Ecological risk assessment has been shown to be a useful tool to characterize the risk of PAHs to organisms and ecosystems [31]. Kalf et al. [32] proposed assessing ecological risk of some organic substances using a risk quotient (RQ) in 1997, and Cao et al. improved the method by considering toxic equivalency factors in 2010. The improved method was demonstrated to be more accurate and scientific [12]. This improved method was used here to assess the ecological risk of PAHs in sediments from the southern part of the Haihe River system.

The levels of risk posed by certain PAHs were characterized using a risk quotient (RQ), which was calculated as 
follows:

$$
\mathrm{RQ}=\frac{C_{\mathrm{PAHs}}}{C_{\mathrm{QV}}},
$$

where $C_{\mathrm{PAHs}}$ was the concentration of certain PAHs in the medium and $C_{\mathrm{QV}}$ was the corresponding quality values of certain PAHs in the medium [32]. In the present study, the negligible concentrations (NCs) and the maximum permissible concentrations (MPCs) of PAHs in water, sediments and bank soil reported by Kalf et al. were used as the values in the medium. MPCs are the concentrations in the environment above which the risk of adverse effects is considered unacceptable to ecosystems, and NCs are the concentrations in the environment below which the occurrence of adverse effects is considered to be negligible [33]. Therefore, $\mathrm{RQ}_{\mathrm{NCs}}$ and $\mathrm{RQ}_{\mathrm{MPCs}}$ were defined as follows:

$$
\begin{gathered}
\mathrm{RQ}_{\mathrm{NCs}}=\frac{C_{\mathrm{PAHs}}}{C_{\mathrm{QV}(\mathrm{NCs})},} \\
\mathrm{RQ}_{\mathrm{MPCs}}=\frac{C_{\mathrm{PAHs}}}{C_{\mathrm{QV}(\mathrm{MPCs})}},
\end{gathered}
$$

where $C_{\mathrm{QV}(\mathrm{NCs})}$ was the values of the NCs of PAHs in the medium and $C_{\mathrm{QV}(\mathrm{MPC})}$ was the values of the MPCs of PAHs in the medium. The $\mathrm{RQ}_{\Sigma \mathrm{PAHs}}, \mathrm{RQ}_{\Sigma \mathrm{PAHs}(\mathrm{NCs})}$ and $\mathrm{RQ}_{\Sigma \mathrm{PAHs}(\mathrm{MPCs})}$ were defined as follows:

$$
\begin{gathered}
\mathrm{RQ}_{\sum \mathrm{PAHs}}=\sum_{i=1}^{n=16} \mathrm{RQ}_{i} \quad\left(\mathrm{RQ}_{\mathrm{i}} \geqslant 1\right), \\
\mathrm{RQ}_{\sum \mathrm{PAHs}(\mathrm{NCS})}=\sum_{i=1}^{n=16} \mathrm{RQ}_{i(\mathrm{NCS})} \quad\left(\mathrm{RQ}_{i(\mathrm{NCS})} \geqslant 1\right), \\
\mathrm{RQ}_{\sum \mathrm{PAHs}(\mathrm{MPCs})}=\sum_{i=1}^{n=16} \mathrm{RQ}_{i(\mathrm{MPC})} \quad\left(\mathrm{RQ}_{i(\mathrm{MPCs})} \geqslant 1\right) .
\end{gathered}
$$

The $R_{\mathrm{N}_{(\mathrm{NCs})}}$ and $R \mathrm{Q}_{(\mathrm{MPCs})}$ of individual PAHs which were not less than 1 were added to calculate the $\mathrm{RQ}_{\Sigma \mathrm{PAHs}(\mathrm{NCS})}$ and the $\mathrm{RQ}_{\Sigma \mathrm{PAHs}(\mathrm{MPC})}$ of total PAHs to fully consider the ecological risk of individual PAHs. The NCs and MPCs of in- dividual PAHs in the sediments are listed in Table 3, and the ecological risk classification is listed in Table 4. An $R Q_{(\mathrm{NCS})}<1.0$ indicated that the single PAHs might be of negligible concern, an $\mathrm{RQ}_{(\mathrm{MPC} S}>1.0$ would indicate that the contamination of the single PAHs posed severe risk, and an $R Q_{(\mathrm{NCS})}>1.0$ and $\mathrm{RQ}_{(\mathrm{MPC})}<1.0$ indicated that the contamination of the single PAHs was of moderate risk.

The mean values of the $R Q_{(\mathrm{NCs})}$ of individual PAHs were all greater than 1.0, and the mean values of $\mathrm{RQ}_{(\mathrm{MPC})}$ of individual PAHs were less than 1.0, except for Nap and Pyr. Nap and Pyr showed a high level of ecological risk, and the other 14 PAHs were at a moderate level of risk. The $\mathrm{RQ}_{\Sigma \text { PAHs(MPCs) }}$ and the $\mathrm{RQ}_{\Sigma \text { PAHs(MPCs) }}$ of the southern part of the Haihe River system are illustrated in Figure 5. The Xidayang reservoir (S2) and the estuary of the Ziya River (S17) showed low ecological risk, Baoding City (S3), Handan City (S11) and Aixinzhuang (S12) showed high ecological risk, and all of the other 19 sites showed moderate risk. The Fuhe River and the Fuyang River were the sections with the highest ecological risk, and they were also the most polluted sections. These data indicated that adverse ecological risk has already been detected in the southern part of the Haihe River system. As industry and transportation develop further, PAH pollution will increase and do harm to the ecosystem and human beings. Therefore, monitoring and controlling of PAHs should be taken into consideration in the southern part of the Haihe River system. Cao et al. [12] assessed the ecological risk of PAHs in water, surface sediments and bank soil of the Luan River and water of the Zhangweinan River with the same method. For the Luan River, the $\mathrm{RQ}_{\Sigma \mathrm{PAHs}(\mathrm{MPC})}$ in water, sediments and bank soil were all zero except for water in Wuliehe (1.53). The mean ecological risk in water was higher than that in sediments and bank soil. Compared with the Luan River, the PAHs pollutants of the southern part of the Haihe River system showed higher ecological risk. For water in the Zhangwei-

\begin{tabular}{|c|c|c|c|c|c|}
\hline PAHs & $\mathrm{NCs}$ & MPCs & PAHs & $\mathrm{NCs}$ & MPCs \\
\hline Nap & 1.4 & 140 & $\mathrm{BaA}$ & 3.6 & 360 \\
\hline Ace & 1.2 & 120 & $\mathrm{Chr}$ & 107.0 & 10700 \\
\hline Acp & 1.2 & 120 & $\mathrm{BbF}$ & 3.6 & 360 \\
\hline $\mathrm{Fl}$ & 1.2 & 120 & $\mathrm{BkF}$ & 24.0 & 2400 \\
\hline Phe & 5.1 & 510 & $\mathrm{BaP}$ & 27.0 & 2700 \\
\hline An & 1.2 & 120 & $\mathrm{DbA}$ & 27.0 & 2700 \\
\hline Flu & 26.0 & 2600 & $\mathrm{InP}$ & 59.0 & 5900 \\
\hline Pyr & 1.2 & 120 & BghiP & 75.0 & 7500 \\
\hline
\end{tabular}
nan River, all sites showed low ecological risk. The mean ecological risk in water was lower than that in sediments of the Zhangweinan River, which was different from the observations of the Luan River.

Table 3 NCs and MPCs of individual PAHs in sediments (ng/g) 
Table 4 Risk classification of individual PAHs and total PAHs

\begin{tabular}{lcc}
\hline & $\mathrm{RQ}_{(\mathrm{NCs})}$ & $\mathrm{RQ}_{(\mathrm{MPCs})}$ \\
\hline Individual PAHs & 0 & \\
Risk-free & $\geqslant 1$ & $<1$ \\
Moderate-risk & & $\geqslant 1$ \\
High-risk & 0 & \\
Total PAHs & $\geqslant 1 ;<800$ & \\
Risk-free & $\geqslant 800$ & 0 \\
Low-risk & $<800$ & 01 \\
Moderate-risk1 & $\geqslant 800$ & $\geqslant 1$ \\
Moderate-risk2 & & \\
High-risk &
\end{tabular}

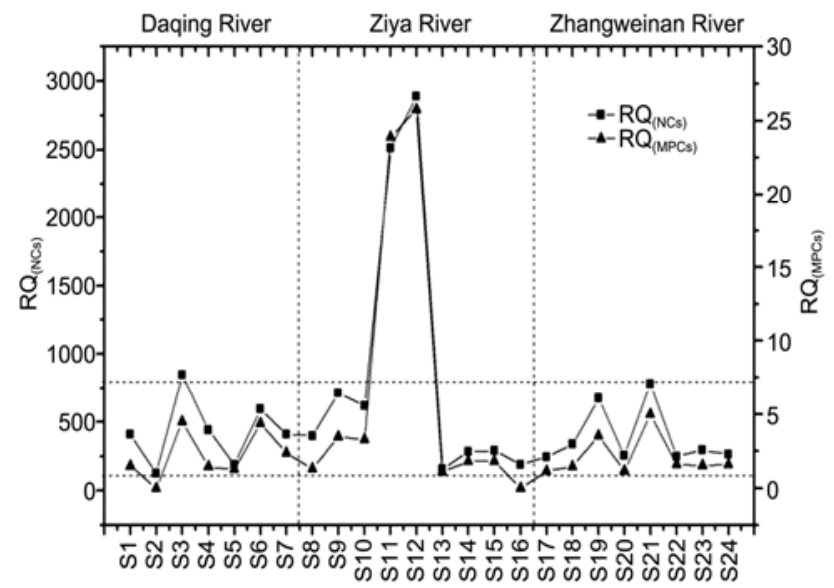

Figure $5 \mathrm{RQ}_{(\mathrm{NCs})}$ and $\mathrm{RQ}_{(\mathrm{MPC})}$ of total $\mathrm{PAHs}$ in sediments from the southern part of the Haihe River system.

\section{Conclusions}

Sixteen polycyclic aromatic hydrocarbons (PAHs) were analyzed in 24 surface sediments sites in 3 rivers of the southern part of the Haihe River system. The concentrations of 16 PAHs ranged from 258.77 to $11296.66 \mathrm{ng} / \mathrm{g}$ dry weight. The composition profile showed that lower molecular weight (2-, 3- and 4-ring) PAHs were predominant in sediments. The PAHs were distributed in accordance with the distribution of cities, and the rural areas and estuaries were less polluted. In the southern part of the Haihe River system, the Fuhe River, the Fuyang River and the Wei River were the most polluted reaches. Ecological risk assessment of 16 PAHs showed that Nap and Pyr were at a high risk level, and the other PAHs were at a moderate level. Ecological risk assessment of the total PAHs at different sites showed that the Xidayang reservoir and the Ziya River estuary were at a low risk level and Baoding, Handan and Aixinzhuang were at a high risk level. Because ecological risk has already been detected in the southern part of the
Haihe River system, more details on the PAHs pollution characteristics in Haihe River Basin, including the presence of PAHs in different mediums and other rivers, should be studied to clarify the behavior, transport and sources of PAHs and to provide suggestions for PAH pollution control.

This work was supported by the National Water Pollution Control Important Specialized Science and Technology of China (2011ZX07203-006) and the National Natural Science Foundation of China (41271496).

1 White K L. An overview of immunotoxicology and carcinogenic polycyclic aromatic hydrocarbons. J Environ Sci Health, 1986, 4: 163-202

2 Heemken O P, Stachel B, Theobald N, et al. Temporal variability of organic micropollutants in suspended particulate matter of the River Elbe at Hamburg and the River Mulde at Dessau, Germany. Arch Enrion Contam Toxicol, 2000, 38: 11-31

3 Binelli A, Sarkar S K, Chatterjee M, et al. A comparison of sediment quality guidelines for toxicity assessment in the Sunderban wetlands (Bay of Bengal India). Chemosphere, 2008, 73: 1129-1137

4 Christensen E R, Irwan A L, Razak A, et al. Sources of polycyclic aromatic hydrocarbons in sediment of the Kinnickinnic River, Wisconsin. J Great Lakes Res, 1997, 23: 61-73

5 Kelly B C, Lkonomou M G, Blair J D, et al. Food web-specific biomagnifications of persistent organic pollutants. Science, 2007, 317: 236-239

6 Guo G H, Wu F C, He H P, et al. Distribution characteristics and ecological risk assessment of PAHs in surface waters of China. Sci China Earth Sci, 2012, 55: 914-925

7 Yang Z F, Feng J L, Niu J F, et al. Release of polycyclic aromatic hydrocarbons from Yangtze River sediment cores during periods of simulated resuspension. Environ Pollut, 2008, 155: 366-374

8 Nobuyasu I, Shuji T, Michio K. Distribution of polycyclic aromatic hydrocarbons in a sediment core from the north basin of Lake Biwa, Japan. Org Geochem, 2010, 41: 845-852

9 Shi Z, Tao S, Pan B, et al. Contamination of rivers in Tianjin, China by polycyclic aromatic hydrocarbons. Environ Pollut, 2005, 134: 97111

10 Jiang B, Zheng H L, Huang G Q, et al. Characterization and distribution of polycyclic aromatic hydrocarbon in sediments of Haihe River, Tianjin, China. J Environ Sci, 2001, 19: 306-311

11 Liu F, Liu J, Chen Q, et al. Pollution characteristics, ecological risk and sources of polycyclic aromatic hydrocarbons (PAHs) in surface sediment from Tuhai-Majia River system, China. Proc Environ Sci, 2012, 13: 1301-1314

12 Cao Z G, Liu J L, Luan Y, et al. Distribution and ecosystem risk assessment of polycyclic aromatic hydrocarbons in the Luan River, China. Ecotoxicology, 2010, 19: 827-837

13 Cao Z G, Liu J L, Wang X M, et al. Pollution characteristics, ecological risk assessment and sources of polycyclic aromatic hydrocarbons (PAHs) in surface water from the Zhangweinan River (in Chinese). Acta Sci Circum, 2010, 30: 254-260

14 Zhou J L, Hong H, Zhang Z, et al. Multi-phase distribution of organic micropollutants in Xiamen Harbour, China. Water Res, 2000, 34: 2132-2150

15 Mai B X, Fu J M, Zhang G, et al. Polycyclic aromatic hydrocarbons in sediments from the Pearl river and estuary, China: Spatial and temporal distribution and sources. Appl Geochem, 2001, 16: 1429_ 1445

16 Guo W, He M C, Yang Z F, et al. Distribution, partitioning and sources of polycyclic aromatic hydrocarbons in Daliao River water system in dry season, China. J Hazard Mater, 2009, 164: 1379-1385

17 Koh C H, Khim J S, Kannan K, et al. Polychlorinated dibenzo-p-dioxins (PCDDs), dibenzofurans (PCDFs), biphenyls (PCBs), and polycyclic aromatic hydrocarbons (PAHs) and 2,3,7,8-TCDD equivalents (TEQs) 
in sediment from the Hyeongsan River, Korea. Environ Pollut, 2004, 132: 489-501

18 State Environmental Protection Administration of China (SEPA). Environmental Quality Standards for Surface Water (GB3838-2002) (in Chinese). 2002

19 Headley J V, Akre C, Conly F M, et al. Preliminary characterization and source assessment of PAHs in tributary sediments of the Athabasca River, Canada. Environ Forensics, 2001, 2: 335-345

20 Zakaria M P, Takada H, Tsutsumi S, et al. Distribution of polycyclic aromatic hydrocarbons (PAHs) in rivers and estuaries in Malaysia: A widespread input of petrogenic PAHs. Environ Sci Technol, 2002, 36: 1907-1918

21 Chen Y Y, Zhu L Z, Zhou R B. Characterization and distribution of polycyclic aromatic hydrocarbon in surface water and sediment from Qiangtang River, China. J Hazard Mate, 2007, 141: 148-155

22 Feng C L, Xia X H, Shen Z Y, et al. Distribution and sources of polycyclic aromatic hydrocarbons in Wuhan section of the Yangtze River, China. Environ Monit Assess, 2007, 133: 447-458

23 Li G C, Xia X H, Yang Z F, et al. Distribution and sources of polycyclic aromatic hydrocarbons in the middle and lower reaches of the Yellow River, China. Environ Pollut, 2006, 144: 985-993

24 Yunker M B, Macdonald R W. Alkane and PAH depositional history, sources and fluxes in sediments from the Fraser River Basin and Strait of Georgia, Canada. Org Geochem, 2003, 34: 1429-1454

25 Fu J, Sheng S, Wen T, et al. Polycyclic aromatic hydrocarbons in surface sediments of the Jialu River. Ecotoxicology, 2011, 20: 940-950
26 Ko F C, Baker J, Fang M D, et al. Composition and distribution of polycyclic aromatic hydrocarbons in the surface sediments from the Susquehanna River. Chemosphere, 2007, 66: 277-285

27 Qiu R Z, Li Y X, Yang Z F, et al. Influence of water quality change in Fu River on wetland Baiyangdian. Front Earth Sci China, 2009, 3: 397-401

28 Zhang J S, Zhang J, Zhang J G, et al. Artificial influence on hydrochemistry of Fuyang River in Handan area. J Hebei Univ Eng (Nat Sci Ed), 2010, 27: 40-45

29 Qin N, Zhu Y, Wu W J, et al. The distributions, composition and their determining factors of polycyclic aromatic hydrocarbons in emergent macrophytes in Small Baiyangdian Lake (in Chinese). J Lake Sci, 2010, 22: 49-56

30 Liu A X, Lang Y H, Xue L D, et al. Probabilistic ecological risk assessment and source apportionment of polycyclic aromatic hydrocarbons in surface sediments from Yellow Sea. Bull Environ Contam Toxicol, 2009, 83: 681-687

31 Wu B, Zhang R, Chen S P, et al. Risk assessment of polycyclic aromatic hydrocarbons in aquatic ecosystems. Ecotoxicology, 2011, 20: 1124-1130

32 Kalf D F, Crommentuijn T, Vandeplassche E J. Environmental quality objectives for 10 polycyclic aromatic hydrocarbons (PAHs). Ecotoxicol Environ Saf, 1997, 36: 89-97

33 Crommentuijn T, Sijm D, Bruijn J D, et al. Maximum permissible and negligible concentrations for some organic substances and pesticides. J Environ Manage, 2000, 58: 297-312

Open Access This article is distributed under the terms of the Creative Commons Attribution License which permits any use, distribution, and reproduction in any medium, provided the original author(s) and source are credited. 